\title{
Direct Determination of Imaging Parameters from Wave Functions in HRTEM
}

\author{
A. Thust, C.L. Jia and K. Urban \\ Institut für Festkörperforschung, Forschungszentrum Jülich GmbH, D-52425 Jülich, Germany
}

The availability of complex-valued electron wave functions, regardless whether acquired by electron holography or by focal-series reconstruction, offers a direct access to information about the object and the optical imaging parameters in use. A part of this information is not contained in single images or can only be extracted tediously from experimental image series using image simulation techniques. In the latter case, however, the knowledge about the object structure is a prerequisite. It is the goal of the present work to explore the information content that can be extracted directly from electron wave functions using as little a-priori knowledge about the object as possible.

Optical aberrations, like defocus, 2-fold astigmatism, and coma can be extracted from crystalline regions. The determination of the even aberrations defocus and 2-fold astigmatism is possible by a numerical minimization of the real part of the wave function following the weak-phase-object approximation (WPOA), even up to thickness values where the WPOA related to the crystal itself does not hold any more. In the case that the constant 3-fold astigmatism is either known or corrected by hardware, the odd aberration coma can be easily extracted from crystalline regions, provided that the object is centrosymmetric and the crystal is not too strongly misaligned. An example for a multiply redundant direct determination of optical parameters is shown in Fig. 2, using a wave function obtained by focal-series reconstruction $[1,2,3]$.

Concerning the determination of the even aberrations it is of crucial importance to check the validity of the underlying WPOA, since a minimum of the real-part contrast is always obtained, regardless whether this minimum is meaningful or not. We present here the phase-histogram method as a means to assess the applicability of the WPOA. According to the WPOA, a wave function is denoted as $\square(\boldsymbol{r})=1+\mathrm{i} \square V(\boldsymbol{r}) t$, with $\square$ the interaction constant, $V(\boldsymbol{r})$ the real-valued crystal potential, and $t$ the object thickness. Thus the function $\square \square 1$ of an ideal WPO is purely imaginary and the phase $\square$ of $\square \square 1$ is either $\square \square$ or $\square$ for all real-space locations. A phase histogram, which displays the frequency of the phase angles $\square$, consists therefore of two delta peaks located at $\square \square$ and $\square$ (Fig. 1). Even in the case of crystalline objects, the filtered amorphous part $\square_{\mathrm{a}}$ of the wave function $\square$, which originates mainly from cover layers on top and bottom of the crystal, can still exhibit these characteristic maxima, albeit in a strongly broadened form. We define here a control parameter $\square$ as the mean squared cosine of the phase angle $\square$, i.e. $\square=<\cos ^{2}(\square)>$, which allows a straightforward check of the compliance of the filtered signal with the WPOA. In the case of an ideal WPO $\square$ is exactly zero, in the case of a random complex object $\square$ is close to $1 / 2$, and in the case of a pure amplitude object $\square$ is exactly 1 . In practice, we succeeded to determine the even aberrations up to values of $\square \approx 0.4$.

\section{References}

[1] W. Coene et al., Ultramicroscopy 64 (1996) 109.

[2] A. Thust et al., Ultramicroscopy 64 (1996) 211.

[3] C.L. Jia and A. Thust, Phys. Rev. Lett. 82 (1999) 5052. 

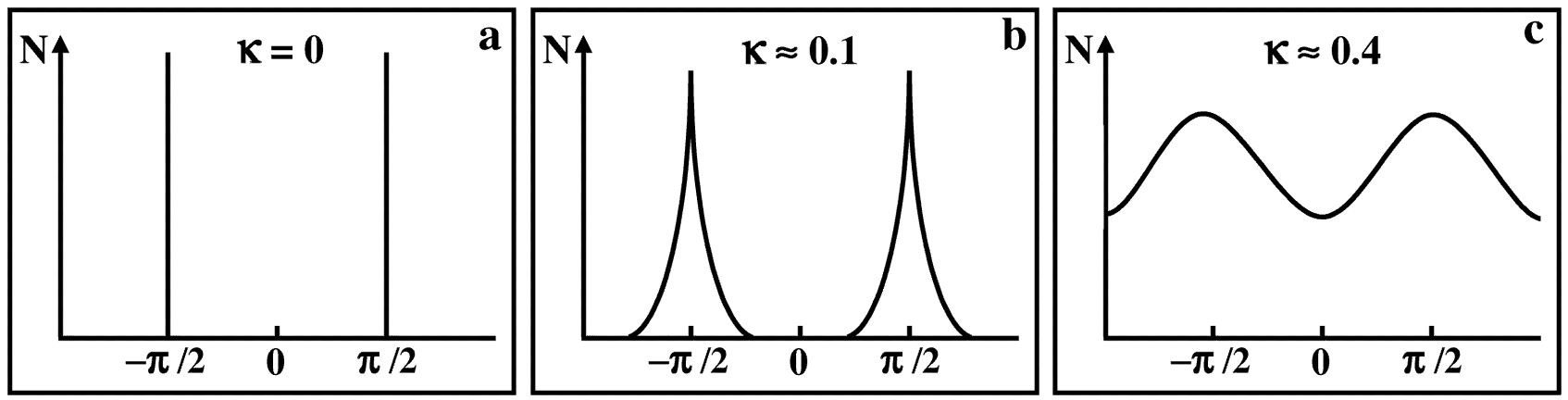

FIG. 1. Schematic phase histograms of $\square a \square$ 1 for the case of (a) an ideal weak-phase object (b) a thin single-layered amorphous object (c) two amorphous cover layers separated by a thin crystal.
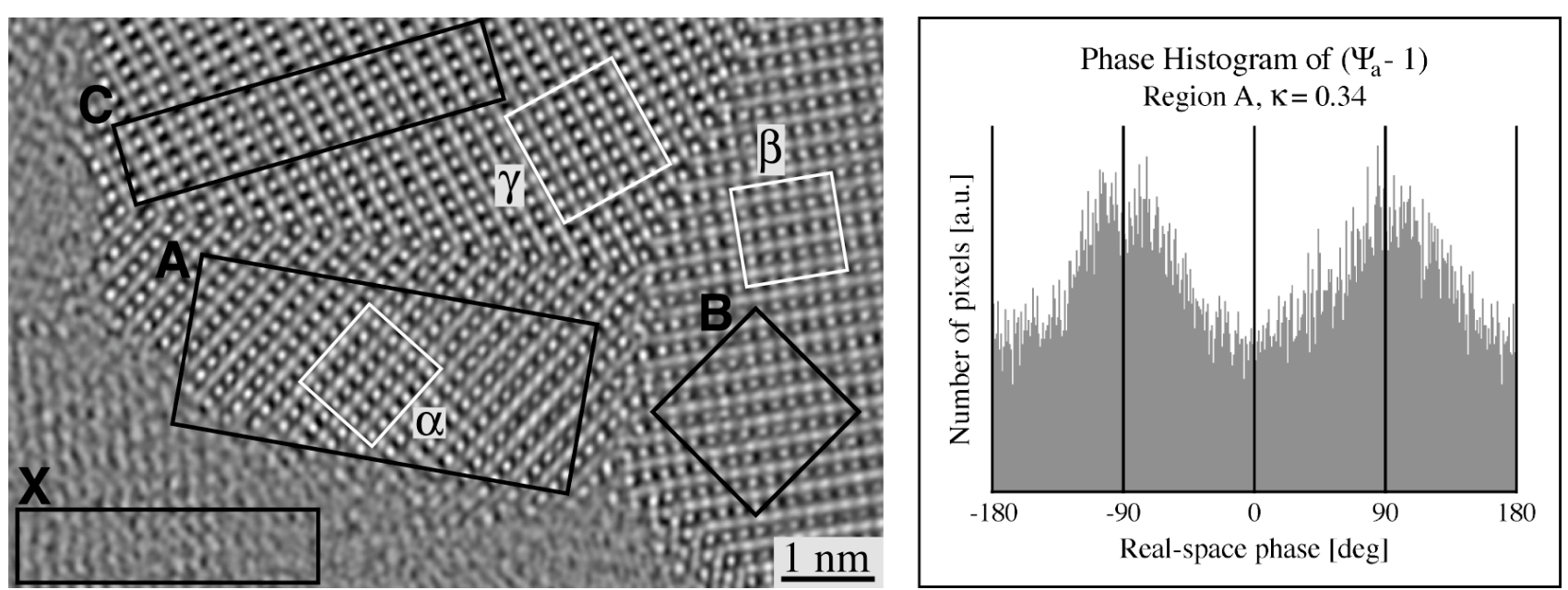

FIG. 2. (Left) Phase of an electron wave function of $<110>$ oriented $\mathrm{BaTiO}_{3}$ containing grain boundaries. The wave function has been corrected numerically for residual lens aberrations. Regions $\mathrm{A}, \mathrm{B}, \mathrm{C}$ and $\mathrm{X}$ were used independently to determine the even aberrations defocus and 2-fold astigmatism, whereas regions $\square, \square$ and $\square$ were used to determine the coma of the objective lens. (Right) Phase histogram obtained from region A after numerical correction of the even aberrations.

TABLE 1. Even aberrations defocus $\square \mathrm{f}_{\mathrm{M}}$ and 2-fold astigmatism $\mathbf{A}_{\mathbf{2}}$ (modulus and azimuth angle $\square$ ) as extracted independently from the regions $\mathrm{A}, \mathrm{B}, \mathrm{C}$ and $\mathrm{X}$ of the wave function of Figure 2. Whereas the defocus may depend on the region of extraction, the 2-fold astigmatism must be independent from the region. From the independent determinations A, B, C and X an error of only $1 \mathrm{~nm}$ in defocus and astigmatism can be concluded.

\begin{tabular}{cccc}
\hline Area & Defocus $\square \mathrm{f}_{\mathrm{M}}[\mathrm{nm}]$ & $\left|\mathbf{A}_{2}\right|[\mathrm{nm}]$ & $\square\left(\mathbf{A}_{2}\right)[\mathrm{deg}]$ \\
\hline $\mathrm{A}$ & 6.0 & 2.7 & 59 \\
$\mathrm{~B}$ & 6.2 & 3.3 & 57 \\
$\mathrm{C}$ & 5.6 & 3.5 & 56 \\
$\mathrm{X}$ & 8.7 & 2.6 & 63 \\
\hline
\end{tabular}

